\title{
Polarizability of 2D and 3D conducting objects using method of moments
}

\author{
Morteza Shahpari ${ }^{1} \quad$ D. V. Thiel $^{2} \quad$ Andrew Lewis ${ }^{3}$
}

(Received 15 November 2012; revised 20 July 2013)

\begin{abstract}
Fundamental antenna limits of the gain-bandwidth product are derived from polarizability calculations. This electrostatic technique has significant value in many antenna evaluations. Polarizability is not available in closed form for most antenna shapes and no commercial electromagnetic packages have this facility. Numerical computation of the polarizability for arbitrary conducting bodies was undertaken using an unstructured triangular mesh over the surface of 2D and 3D objects. Numerical results compare favourably with analytical solutions and can be implemented efficiently for large structures of arbitrary shape. Keywords: computational methods, singular integral equations, moment methods, polarizability
\end{abstract}

http://journal . austms.org.au/ojs/index.php/ANZIAMJ/article/view/6405 gives this article, (c) Austral. Mathematical Soc. 2013. Published August 16, 2013, as part of the Proceedings of the 16th Biennial Computational Techniques and Applications Conference. ISSN 1446-8735. (Print two pages per sheet of paper.) Copies of this article must not be made otherwise available on the internet; instead link directly to this URL for this article. 


\section{Contents}

1 Introduction

C447

2 Formulation

C448

3 Implementation

C452

4 Validation

C452

5 Discussion and conclusion

C455

References

C456

\section{Introduction}

Polarizability is an important parameter in a variety of physical science disciplines including scattering and molecular and chemical physics. Recently engineers used this parameter for antenna modelling. Gustafsson et al. [6] demonstrated the relationship between the maximum possible antenna gainbandwidth product and the polarizability of the antenna obstacle. This is now recognised as a method of calculating a fundamental limit for antennas. The maximum electromagnetic scattering of a plane wave incident on an obstacle (e.g., metamaterial [10, 11], periodic arrays [7]) is related to the static polarizability of the obstacle. Popular commercial packages for antenna modelling, for example, ANSYS HFSS, FEKO, AWR and IE3D, do not calculate the polarizability.

In this article we use the method of moments (MOM) to calculate the polarizability of arbitrary geometries. The MOM technique is commonly used in modelling wire structures for both radiation and scattering problems, assuming a thin wire approximation $[2,8]$. In the MOM polarizability calculation, three dimensional structures are modelled using triangular mesh elements. 
The technique was implemented in Matlab to calculate the polarizability of arbitrary shaped objects with infinite conductivity (i.e. perfect electric conductors, PEC). FEKO, which is an antenna simulation package, was used to create triangular mesh elements for arbitrary objects and the 3D mesh was exported to the MOM routine in Matlab using the STL format ${ }^{1}$. Triangular mesh elements have the advantage of constructing arbitrary geometries without staircase approximations.

A brief description of the MOM solution is described in Section 2. Section 3 explains the implementation of the algorithm. Section 4 demonstrates the validity of the code by comparing the MOM results with closed form solutions of simple geometric objects.

\section{Formulation}

In electromagnetics, Laplace's equation is used to describe the electrostatic potential in a charge free region. Assuming that the antenna has no static charge, then Laplace's equation in integral form is [8]

$$
x_{j}+C_{j}=\iint_{S} \frac{\rho_{j}\left(x^{\prime}\right)}{4 \pi\left|x-x^{\prime}\right|} d S^{\prime},
$$

where $\rho_{j}$ and $x_{j}$ are the surface charge density and the distance from the origin, respectively, along the $x_{j}$ axis when the object is located in a static field with unit amplitude in the $\hat{x}_{j}$ direction (see Figure 1). The integral is over the surface of the geometry $\mathbf{S}$. Also, $\boldsymbol{x}$ and $\boldsymbol{x}^{\prime}$ are observation and source points, respectively. If the object is asymmetrical or is offset from the origin, then the sum of the total induced charge tends to be nonzero. This is contrary to the charge conservation law. The constant $C_{j}$ is added to ensure

\footnotetext{
${ }^{1}$ Standard tessellation language (STL) is the industrial standard for handling triangulated meshes http://wiki.netfabb.com/STL_Files_and_Triangle_Meshes, http: //en.wikipedia.org/wiki/STL_\%28file_format\%29.
} 


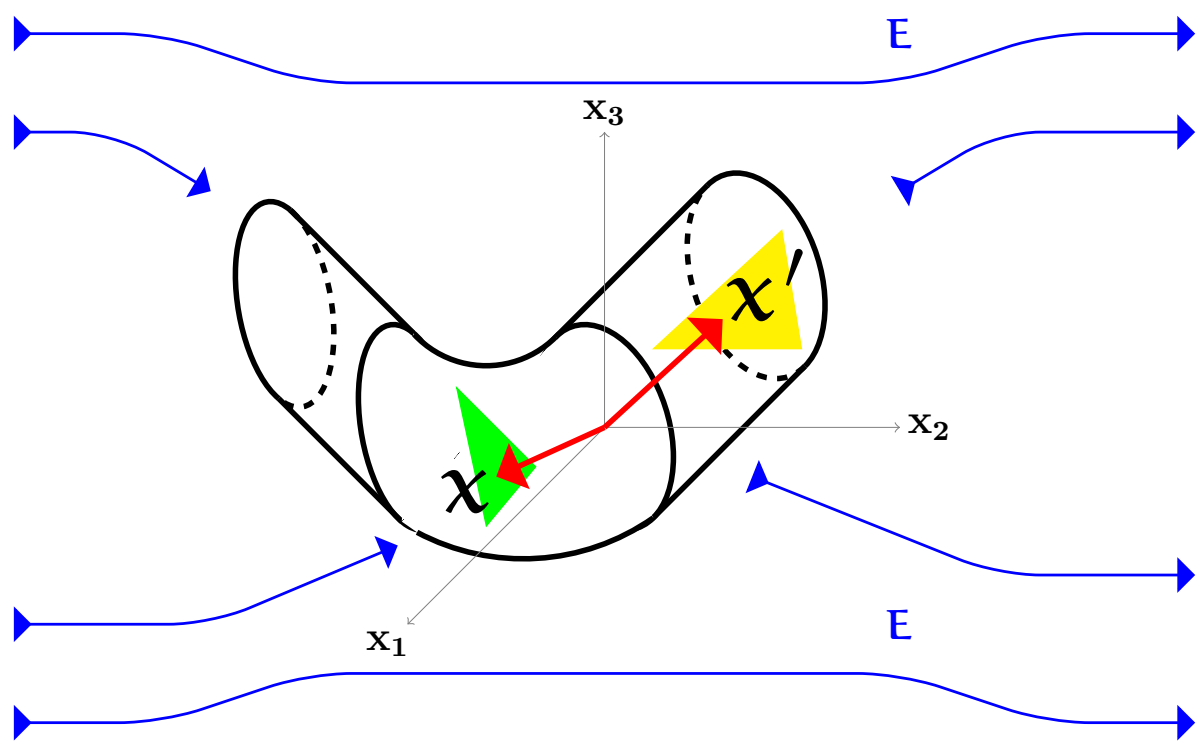

Figure 1: A wire antenna with arbitrary shape located in an applied electric field $\mathbf{E}$ oriented in the $\hat{x}_{2}$ direction. Position of source and observation mesh are denoted by $\boldsymbol{x}^{\prime}$ and $\boldsymbol{x}$.

the total charge on the object is zero:

$$
\iint_{S} \rho_{j}(x) d S=0 .
$$

After finding $\rho_{j}$ over $S$, the polarizability $\gamma_{i j}$ in the $\hat{x}_{i}$ direction due to the applied field in the $\hat{x}_{j}$ direction is

$$
\gamma_{i j}=\iint_{S} x_{i} \rho_{j}(x) d S
$$

The polarizability $\gamma_{i j}$ has units $\mathrm{m}^{2} \mathrm{~V}^{-1}$. However, it is common to use the normalised polarizability $\gamma_{i j} / a^{3}$ which has units $\mathrm{m}^{-1} \mathrm{~V}^{-1}$ for a the radius of the smallest surrounding sphere. 
The aim of the MOM method is to convert (1) to the standard matrix form $\mathrm{L} \rho_{j}=\mathrm{g}$ using a discrete mesh and the summation of basis functions. Because of their simplicity, pulse functions $f_{n}$ are used for both basis and testing functions:

$$
f_{n}= \begin{cases}1 & \text { on } \Delta S_{n} \\ 0 & \text { otherwise }\end{cases}
$$

This choice of basis and testing functions yields the following double integral over the mesh elements for the matrix elements [1]

$$
\mathrm{L}_{\mathrm{mn}}=\frac{1}{4 \pi} \iint_{A_{m}}\left(\iint_{A_{n}} \frac{1}{\left|x-x^{\prime}\right|} \mathrm{d} S\right) \mathrm{d} S^{\prime}
$$

where $A_{m}$ and $A_{n}$ are the areas of the mth and nth triangular mesh (source and observation mesh), respectively. For non-diagonal elements

$$
\mathrm{L}_{\mathrm{mn}} \approx \frac{1}{4 \pi} \frac{\mathrm{A}_{\mathrm{n}} \mathrm{A}_{\mathrm{m}}}{\left|\boldsymbol{x}-\boldsymbol{x}^{\prime}\right|}
$$

The matrix L shows singular behaviour on the diagonal elements. This is because the denominator in (1) goes to zero when $\boldsymbol{x}$ approaches $\boldsymbol{x}^{\prime}$. For diagonal elements, an exact solution to the integral in (5) was obtained by Eibert and Hansen [3, eq. (25)]:

$$
\begin{aligned}
L_{n n}= & \frac{A_{n}^{2}}{4 \pi}\left\{\frac{1}{6 \sqrt{a}} \log \left[\frac{(a-b+\sqrt{a} d)(b+\sqrt{a} \sqrt{c})}{(-b+\sqrt{a} \sqrt{c})(-a+b+\sqrt{a} d)}\right]\right. \\
& +\frac{1}{6 \sqrt{c}} \log \left[\frac{(b+\sqrt{a} \sqrt{c})(-b+c+\sqrt{c} d)}{(-b+\sqrt{a} \sqrt{c})(b-c+\sqrt{c} d)}\right] \\
& \left.+\frac{1}{6 d} \log \left[\frac{(a-b+\sqrt{a} d)(-b+c+\sqrt{c} d)}{(b-c+\sqrt{c} d)(-a+b+\sqrt{a} d)}\right]\right\},
\end{aligned}
$$

where $d=\sqrt{a-2 b+c}$ and $a, b$ and $c$ are computed from position vectors 


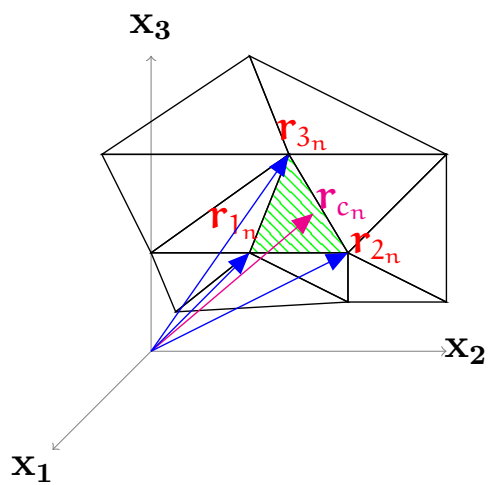

Figure 2: Discretization of the 3D conductor into mesh elements. The calculation of $l_{n n}$ needs $\boldsymbol{r}_{1_{n}}, \boldsymbol{r}_{2_{n}}$ and $\mathbf{r}_{3_{n}}$, the positions of the three vertices of the $n$th mesh element, and $\mathbf{r}_{\mathfrak{c}_{n}}$ points to the centre of of the mesh.

of mesh vertices $\boldsymbol{r}_{1}, \boldsymbol{r}_{2}$ and $\boldsymbol{r}_{3}$ (see Figure 2) by

$$
\begin{aligned}
& a=\left(\mathbf{r}_{3}-\mathbf{r}_{1}\right) \cdot\left(\mathbf{r}_{3}-\mathbf{r}_{1}\right), \\
& \mathbf{b}=\left(\mathbf{r}_{3}-\mathbf{r}_{1}\right) \cdot\left(\mathbf{r}_{3}-\mathbf{r}_{2}\right), \\
& c=\left(\mathbf{r}_{3}-\mathbf{r}_{2}\right) \cdot\left(\mathbf{r}_{3}-\mathbf{r}_{2}\right) .
\end{aligned}
$$

The mth element of $\mathrm{g}$ is

$$
g_{m}=\left(x_{j_{m}}+C_{j}\right) A_{m},
$$

where $x_{j_{m}}$ is the projection of the centre of the $m$ th mesh along the $x_{j}$ axis. As long as the centre of the geometry is located at the coordinate origin, $C_{j}$ is zero. Problems arise when the object is shifted from the origin and large errors can result, particularly for complicated shapes. As far we know, there is no published literature which describes how to calculate $C_{j}$ in general. To find $C_{j}$ we define a complementary parameter $u_{m}=x_{j_{m}} A_{m}$ and rewrite $g$ as

$$
g=u+C_{j} A .
$$


The induced charge $\rho_{j}$ on the object is calculated from $\mathrm{L}^{-1} \mathrm{~g}$, or,

$$
\rho_{j}=L^{-1} u+C_{j} L^{-1} A .
$$

In (11) $\rho_{j}, \mathfrak{u}$ and $A$ are vectors of dimension equal to the number of meshes while $\mathrm{L}$ is the MOM matrix. By the charge conservation law, the sum of the induced charge $\rho_{j}$ has to vanish. Therefore $C_{j}$ is

$$
C_{j}=-\frac{\sum L^{-1} u}{\sum L^{-1} A}
$$

Finally the polarizability is computed from

$$
\gamma_{i j}=\left(x_{i_{1}} \ldots x_{i_{m}} \ldots x_{i_{n}}\right) L^{-1} g .
$$

where $x_{i_{n}}$ is the position of the centre of the $n$th mesh element in the $x_{i}$ direction, that is $\mathbf{r}_{c_{n}} \cdot \hat{x}_{i}=x_{i_{n}}$.

\section{Implementation}

The complete solution was implemented in Matlab code by substituting equations (6)-(12) into (13) (see Figure 3). The calculated 3D polarizability is normalised by $a^{3}$, where $a$ is the smallest radius of the sphere that encloses the object. The mesh file geometry is imported into the Matlab program. We used the MOM simulation package [4] to generate the triangular mesh on the 3D objects. Many other commercial and non-commercial packages can also be used for this mesh generation (e.g., AUTOCAD and ANSYS).

\section{Validation}

The code was validated by comparing the computed results with several published closed form values for simple 3D geometric shapes including a 
Triangular mesh file

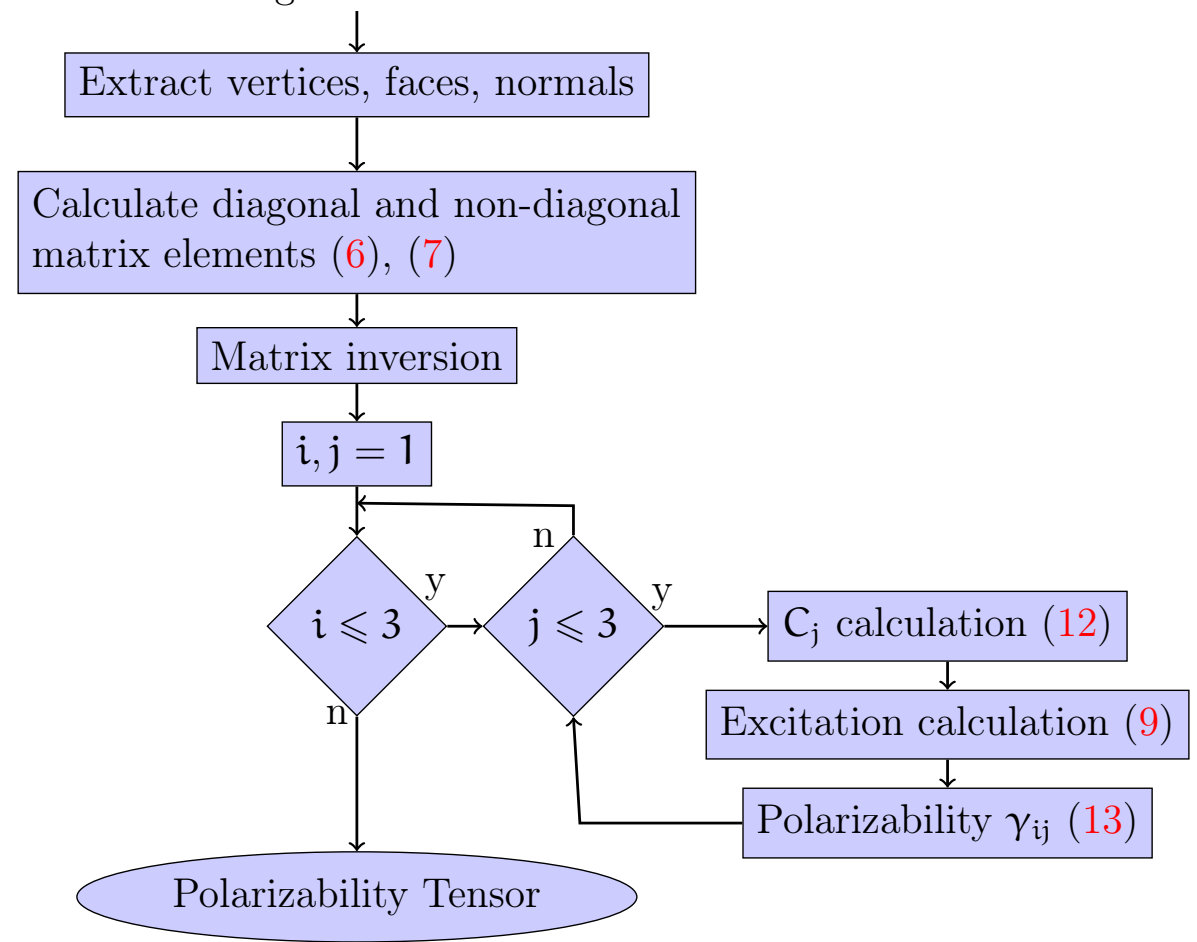

Figure 3: Program flowchart for the polarization calculation of an arbitrary conductor shape using an array of triangular mesh elements.

sphere, circular disk, and toroidal ring [6]. Results are normalised by $\mathrm{a}^{3}$ and shown in Table 1.

Figure 4 shows the polarizability of spheroids of semi-axes $a_{x}, a_{y}$ and $a_{z}$ in terms of different aspect ratios. For the oblate and prolate geometries with circular cross sections in $x y$-plane, the red and blue lines illustrate the analytical behaviour of the tangential and perpendicular polarizabilities, respectively, relative to the $x y$-plane [9]. Numerical results were computed for three different aspect ratios (1,2 and 4). There is strong agreement with 
Table 1: Comparison of the numerical results with those of Gustafsson et al. [6]

\begin{tabular}{cccc} 
Geometry & Sphere & Disk & Toroid \\
\hline Analytic result & 12.56 & 5.27 & 2.64 \\
Numerical result & 12.59 & 5.22 & 2.63
\end{tabular}

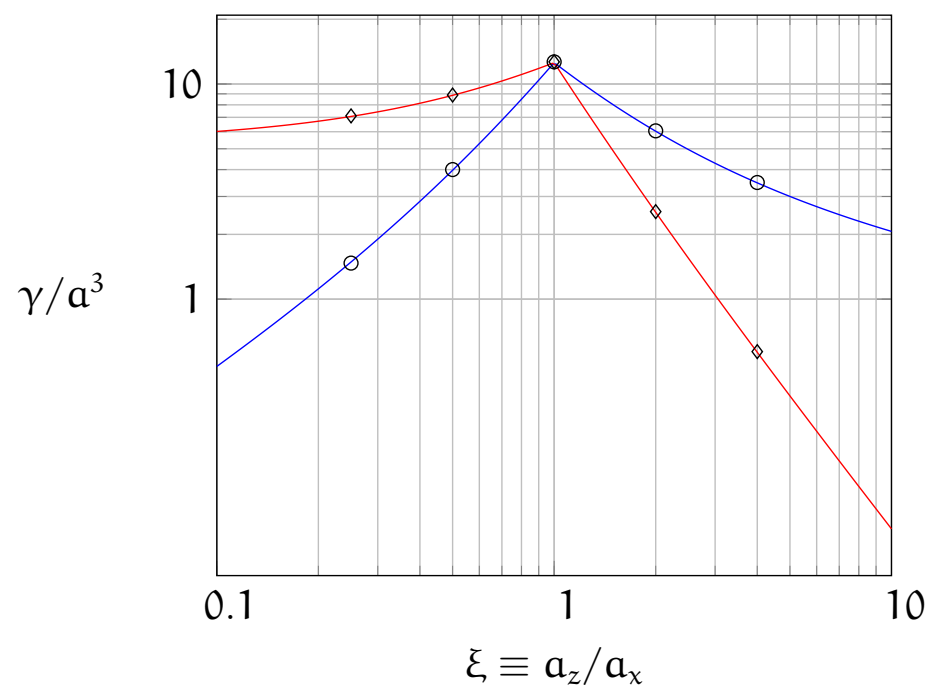

Figure 4: Analytic tangential (red line) and perpendicular (blue line) polarizability of spheroids with different aspect ratios $\xi=a_{z} / a_{x}$. Diamonds and circles refer to our numerical results. When $a_{x}=a_{z}$ the object is a sphere.

analytical expressions. A sphere $\left(a_{x}=a_{z}\right)$ has the highest polarizability and $\gamma_{\mathrm{h}}=\gamma_{v}$ at this point. A spheroid with an aspect ratio $\xi=\mathrm{a}_{z} / \mathrm{a}_{\mathrm{x}}=2$ has a different shape to a spheroid with an aspect ratio of $\xi=0.5$, and so Figure 4 is not symmetric about the vertical line $a_{x}=a_{z}$.

Figure 5 shows the polarizability of several infinitely thin (i.e., 2D), perfectly conducting rectangles with different aspect ratios. These results are compared to those of Gustafsson [5]. When the aspect ratio is unity the object is square. The two sets of results show good agreement. 


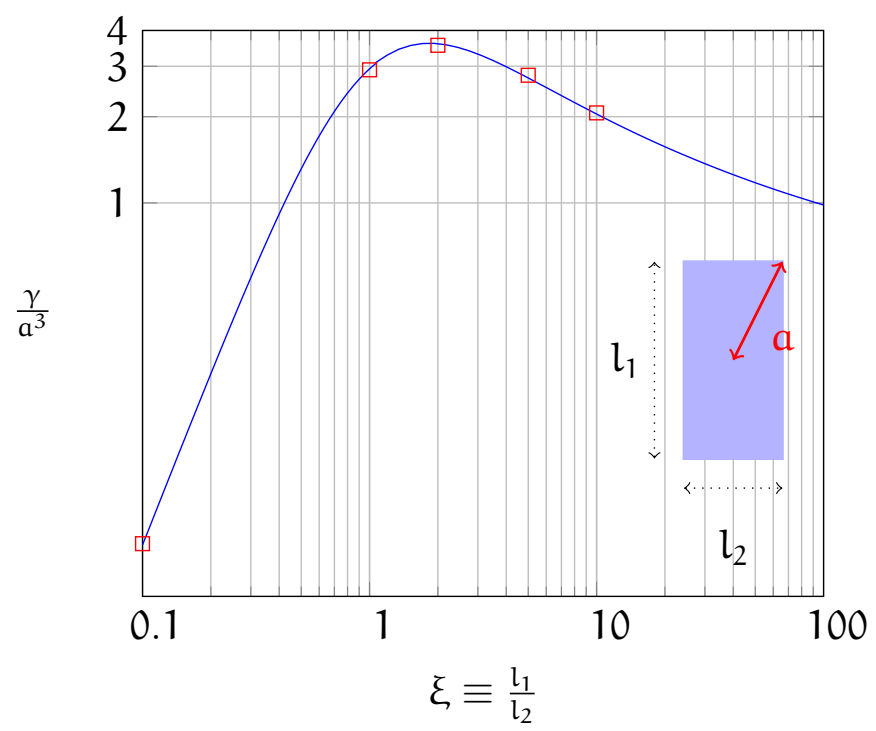

Figure 5: Normalised vertical polarizability of infinitely thin rectangles with different length to width ratios (continuous line). The rectangles indicate the aspect ratio corresponding to the calculated values (red squares). An aspect ratio of unity is square.

\section{Discussion and conclusion}

A numerical technique to compute the polarizability of conducting bodies with arbitrary shapes was reported. A desktop computer with Intel@core i5 $\mathrm{CPU}$ and $4 \mathrm{~GB}$ of RAM was used for the numerical calculations. The program is fast (less than a minute) for geometries with less than 3000 mesh elements. However, the calculation time increases with the cube of mesh elements. For example, the computational time is 4 and 14 minutes for geometries with 6000 and 7000 mesh elements, respectively. In addition to the Matlab and 
STL code, available as supplementary material, ${ }^{2}$ a GUI interface is available from the authors.

The utilisation of other basis and test functions (e.g., RWG basis functions) is recommended to improve this technique and obtain more accurate results. This investigation used the library Matlab functions, but faster and more efficient methods maybe available for the matrix inversion.

Acknowledgements Morteza Shahpari appreciates the Griffith Postgraduate Research Scholarship from Griffith University. This work is partly funded by Australian Research Council grant DP130102098.

\section{References}

[1] P. Arcioni, M. Bressan, and L. Perregrini. On the evaluation of the double surface integrals arising in the application of the boundary integral method to 3D problems. IEEE Trans. Microw. Theory Tech., 45(3):436-439 (1997). doi:10.1109/22.563344 C450

[2] G. J. Burke and A. J. Poggio. Numerical Electromagnetics Code (NEC) - Method of Moments. National Technical Information Service, U.S. Department of Commerce (1981). http://www.ntis.gov/search/product. aspx?ABBR=ADA956129 C447

[3] T. F. Eibert and V. Hansen. On the calculation of potential integrals for linear source distributions on triangular domains. IEEE Trans. Antennas Propag., 43(12):1499 -1502 (1995). doi:10.1109/8.475946 C450

[4] FEKO, EM Software and Systems. FEKO 6.1.1, 1998-2011. http://www.feko.info/ C452

${ }^{2}$ http://anziamj.austms.org.au/ojs/index.php/ANZIAMJ/article/ downloadSuppFile/6405/1293 
[5] M. Gustafsson. Physical bounds on antennas of arbitrary shape. Loughborough Antennas and Propagation Conference, (2011). doi:10.1109/LAPC.2011.6114002 C454

[6] M. Gustafsson, C. Sohl, and G. Kristensson. Illustrations of new physical bounds on linearly polarized antennas. IEEE Trans. Antennas Propag., 57(5):1319 -1327 (2009). doi:10.1109/TAP.2009.2016683 C447, C453, C454

[7] M. Gustafsson, C. Sohl, C. Larsson, and D. Sjöberg. Physical bounds on the all-spectrum transmission through periodic arrays. Europhysics Letters, 87(3):34002 (2009). doi:10.1209/0295-5075/87/34002 C447

[8] R. F. Harrington. Field Computation by Moment Methods. IEEE Press (1993). C447, C448

[9] R. E. Kleinman and T. B. A. Senior. Rayleigh scattering. In V. V. Varadan and V. K. Varadan, editors, Handbook on Acoustic, Electromagnetic and Elastic Wave Scattering, volume 2, pages 1-70. Elsevier (1986). C453

[10] C. Sohl, M. Gustafsson, and G Kristensson. Physical limitations on broadband scattering by heterogeneous obstacles. J. Phys. A: Math. Theor., 40(36):11165 (2007). doi:10.1088/1751-8113/40/36/015 C447

[11] C. Sohl, C. Larsson, M. Gustafsson, and G. Kristensson. A scattering and absorption identity for metamaterials: Experimental results and comparison with theory. J. Appl. Phys., 103(5):054906 (2008). doi:10.1063/1.2875727 C447

\section{Author addresses}

1. Morteza Shahpari, Centre for Wireless Monitoring and Applications, School of Engineering, Griffith University, Queensland 4111, Australia. mailto:m.shahpari@griffith.edu.au 
2. D. V. Thiel, Centre for Wireless Monitoring and Applications, School of Engineering, Griffith University, Queensland 4111, Australia. mailto:d.thiel@griffith.edu.au

3. Andrew Lewis, Institute for Integrated and Intelligent Systems, Griffith University, Queensland 4111, Australia. mailto:a.lewis@griffith.edu.au 